\title{
BUILDING ON DESIGN THINKING TO WORK CREATIVELY WITH KNOWLEDGE: A TALE OF TWO PARADIGMS
}

\author{
Paul J. McELHERON \\ Associate Professor - VIA Design \& Business, Herning, Denmark
}

\begin{abstract}
We began to explore design thinking initially as a means of creating a common language between design and business students. Subsequent studies using randomized control trials involving several hundred students working in multidisciplinary teams confirmed that teams following a design thinking pedagogy do indeed generate more innovative design concepts relative to teams that do not. Observations allowed us to suggest how and why this is the case. However, these innovations are generally incremental in nature. Observations made on the most effective teams in terms of radical innovative concept development revealed they applied an effective knowledge acquisition, communication and idea development strategy and this aligns with the principles of a knowledge building pedagogy. Our challenge as educators then became to explore how, what a small number of teams do intuitively, maybe made explicit for all student teams. We integrated knowledge building principles with the design thinking process to develop a combined approach which capitalizes on the advantages of both pedagogies. Subsequent studies suggest that teams using this combined approach are encouraged to close knowledge gaps and engage in sustained idea development resulting in a multiplier effect where subsequent knowledge acquisition encourages teams to be creative with knowledge. This paper reports on one such study and the pedagogical methods employed to achieve this. This combined approach is relevant to education because capacities for creative knowledge work are in high demand by the industries we serve and while, in our experience, creativity comes naturally to most students, the sustained development of ideas does not and requires support.
\end{abstract}

Keywords: Design thinking, knowledge building, creative problem solving, pedagogy

\section{INTRODUCTION}

The expectations concerning the competencies required by our students are expanding. The need for Tshaped skills, a metaphor for the depth and breadth of an individual's skills resonates within many industries. Creative problem solving has been identified as an important $21^{\text {st }}$ Century skill [1] and design thinking, (a human centred creative problem solving process), has found application in a range of sectors including education driven by its promise as a driver of innovation and an aid to creative problem solving.

As a design and business school, we are aware that design and business students take different approaches to problem solving. Studies carried out on our own students suggest design students make much use of visualization and prototyping and generally adopt an abductive thinking approach. Business students tend to adopt a more analytical, deductive approach and rarely use visualization or prototyping. Expert problem solvers, including designers frequently use their experience and knowledge to solve problems but students have relatively little in terms of experience or domain knowledge [2]. However, design and business students are frequently required to work together in multi-disciplinary teams to solve real world problems as part of the curriculum and, given that design and business students work differently it was felt that some form of common language and understanding between the design and business disciplines may facilitate this.

This led us to explore design thinking as a vehicle for achieving this common understanding. We were aware of the growing body of literature on design thinking and several design thinking workshops, (of varying quality), had been conducted at our institution by external consultants. We were also aware of the criticism surrounding design thinking. The term itself had been called confusing and conflicting [3], 
and a public relations term for good old-fashioned creative thinking [4]. Human centred design has also attracted criticism for lacking the skills and knowledge needed to generate radical innovation [5]. Our own reservations included that the process was "light on theory" and there appeared to be little in terms of empirical evidence that it "worked". Most accounts of the success of design thinking were anecdotal, reflective or based on small-scale case studies.

However, we could see the possible potential with design thinking to combine the design and business approaches to creative problem solving and communicate the value to students. We developed a prototype model comprising of the main elements of design thinking, (empathy for user needs, problem framing to establish real need, idea generation, prototyping and concept testing). This model was introduced to first semester students in the form of 2-3-day workshops. Typically, a cohort of 80 or so design and business students working in multidisciplinary teams were presented with a real-world design challenge and asked to develop innovative solutions.

Following a series of these workshops, there was a consensus from teaching staff and students that they did help build a common language and understanding between the design and business disciplines. The concepts generated featured largely incremental innovations, improvements to existing systems although they were "useful" with many of the ideas generated being implemented.

\section{BUILDING ON DESIGN THINKING}

Encouraged by these early results, we decided to explore design thinking processes and practices and their role in student innovation in more detail. Specifically: To what extent do they work, (quantitative, suggestive)? How do they work, (qualitative)? Why do they work, (theoretical, explanatory)? The approach to answering these questions was to run a series of empirical studies comparing the innovative concepts from multi-disciplinary student teams using design thinking with similar teams who did not. A mixed methods research approach was adopted, using randomized control trials and including quantitative analysis of design teams' concepts, observations, questionnaires and interviews. Data was collected from multiple studies involving over 400 design and business students at various stages of their education.

Scores were assigned to team concepts using Nilsson's Taxonomy of Creative Design [6] or Guilford's [7] measures of fluency, flexibility, originality and elaboration plus relevance. The concept scores from the design thinking teams in the test group scored, on average $80 \%$ higher than the average scores from the non-design thinking control group [8]. Observations and interviews allowed suggestions to be made as to how and why this is the case. The focus on understanding user needs is helpful in many instances, especially if it involves effective methods to discover these needs, for example observation or experimentation as opposed to merely asking them. Framing problems in new and interesting ways assists in finding the "right" problem to solve. Problem finding ability and effort has been found to predict creative accomplishments above and beyond other known predictors such as divergent thinking and intelligence [9]. The idea generation phase and associated practices facilitate the generation and combination of ideas. Visualisation techniques aid communication of ideas and helps ensure ideas do not get "lost". The construction of prototypes is something that engages students and assists the communication and refinement of ideas. Concept testing helps capture user input which may then be used to further refine concepts. Overall, our conclusion is that the design thinking process delays teams jumping directly into "solution mode" and helps overcome creative blocks. The process is inclusive and encourages teamwork and collaboration. Students frequently reported an increase in their creative confidence [10].

\subsection{Introducing knowledge building}

Returning to the question of how innovative were the concepts generated, design thinking teams' concepts scored, on average, higher than non-design thinking teams concepts in terms fluency, (number of ideas), flexibility, (range of ideas), combinations of ideas and to some extent originally, (the unusualness of the concept). Radical innovation was elusive, but it did sometimes occur. Observations made on teams with the highest innovation scores working on problems that required both creativity and knowledge to reach a solution often revealed they adopted an effective knowledge acquisition and sharing strategy within a design thinking framework.

As an example, we observed two teams over five days applying design thinking to a problem sponsored by a company the produces apps: to design an app for people diagnosed with ADHD/Autism (AUT) with a means of monitoring, and providing feedback on their emotions thereby reducing anxiety in their 
daily lives. Both teams did succeed in developing concepts that the sponsoring company considered innovative and feasible in terms of functionality and cost. Team one followed the design thinking process and, focusing on empathy for user needs, (translated into "ease of use"), developed a concept which contained several novel elements, for example how emotions could be linked to certain events and represented in a form understandable to people with the ADHD/AUT condition. However, team two's concept was far more sophisticated combining several bio-monitoring features and an emotional feedback and anxiety reduction system that could prevent anxiety reaching problem levels. The team demonstrated a deep understanding of the ADHD-Autism-Anxiety cycle and how this might be disrupted. Team two were engaged in what Engle \& Conant describe as research - share - perform cycles where students collaboratively construct meaning and action transforming the classroom into a community of discourse." [11]. The team's focus was on identifying and closing gaps in their knowledge by consulting expert sources of information then using this knowledge to formulate problems at a deeper level. Design thinking was being applied in such a way that the team was being creative around their improved ideas rather than their first ideas. The discourse was more than just knowledge sharing, it had more to do with critiquing, constructing \& refining this knowledge. They continued to reflect on what they knew, what were their most promising ideas, what they need to know \& what action plan they would use to move forward \& this action was often further research.

This method of working had also been observed in highly effective teams in our other design thinking studies. How might this be explained, was it a result of individual strengths, team composition, and communication etc? A search of the various approaches to knowledge creation capacity suggested that the methods used by these very effective teams shared several similarities with the principles of a concept known as knowledge building, [12]. Knowledge building is a theory of community learning based on research dating back to the 1970's by Carl Bereiter and Malene Scardamalia of the Ontario Institute for Studies in Education (OISE), (University of Toronto) and has been defined as, the advancement of knowledge as a result of sustained collaborative development of ideas, [13]. Knowledge building has its origins in how knowledge creating organisations generate ideas. The research has been conducted in educational settings and the process of knowledge creation is by collaborative effort by groups of students who produce and improve their epistemic artifacts mediated via a knowledge building discourse using appropriate epistemic criteria to improve ideas. The aim is to advance the frontiers of knowledge as perceived by participants.

Scardamalia \& Bereiter, [13] have identified several important strategic differences or themes that support the concept of knowledge building. These include: sustained idea improvement, emergent understanding as a community as a primary and sustaining goal, encouragement of emergent goals and products to support understanding, constructive use of authoritative sources of information, the application of declarative and procedural knowledge and the importance of operating in design mode, (the mode of invention and idea development). These themes have been elaborated upon to form a set of 12 knowledge building principles designed to be used as pedagogical "scaffolds".

Our studies suggested that combining the principles of knowledge building and process and practices of design thinking might help student teams work creatively with knowledge. However, while there are some similarities between the constructivist design thinking and knowledge building pedagogies, there are important differences as Table 1 indicates:

Table 1. Similarities and differences - design thinking and knowledge building

\begin{tabular}{|l|l|}
\hline \multicolumn{1}{|c|}{ Design Thinking } & \multicolumn{1}{c|}{ Knowledge Building } \\
\hline No agreed definition & Has an agreed definition \\
\hline $\begin{array}{l}\text { Origins: Stanford "Creative thinking" - 1950s, } \\
\text { IDEO - 2000s and design methods discourse }\end{array}$ & $\begin{array}{l}\text { Origins: 1970s: How knowledge creating } \\
\text { organisations "create" knowledge }\end{array}$ \\
\hline Has a clear process & Eschews a process - principle based \\
\hline Generally incremental innovation? & Sometimes more radical innovation? \\
\hline Mindset complicated & Mindset clearly defined \\
\hline $\begin{array}{l}\text { Aim: a better solution, outcome often an object } \\
\text { or artifact }\end{array}$ & $\begin{array}{l}\text { Aim: sustained idea development and } \\
\text { advancement of knowledge }\end{array}$ \\
\hline $\begin{array}{l}\text { "Evidence" largely anecdotal or case study } \\
\text { based }\end{array}$ & A more robust empirical evidence base \\
\hline Implementation - relatively straightforward & Implementation - challenging \\
\hline
\end{tabular}


Integrating knowledge building principles within a design thinking process faced three challenges as we saw them. Firstly, the principles, there are 12 of them, a large number for practical purposes. Several of the knowledge building principles are common to design thinking, (democratization of knowledge, idea diversity, real ideas, authentic problems and building on the ideas of others). This allowed us to condense and combine the knowledge building principles into five core concepts we considered critical to implementing knowledge building in a creative problem solving context, these are: Ideas are considered improvable rather than being simply accepted or rejected. Facilitate epistemic agency empower learners to set goals, plan and assess their own progress. Maintain a knowledge building discourse - not just sharing ideas but aim at transforming and advancing knowledge. Rise-above - work with diverse ideas in complex problem spaces and formulate problems at higher levels. Make constructive use of, and critically evaluate expert sources of information. Secondly, we had to address the (lack of) process issue. Design thinking has a very clear process, knowledge building is entirely principle based and eschews a process as it is believed that adhering to a process interferes with knowledge creation, There is no order or structure to the principles but in our experience novice designers value a process highly. Scardamalia \& Bereiter acknowledge that adhering to a principle based rather than a procedural approach has undoubtedly impeded the spread of knowledge building [14]. To address this, we modified our design thinking model to incorporate knowledge building principles and practices, (Figure 1).

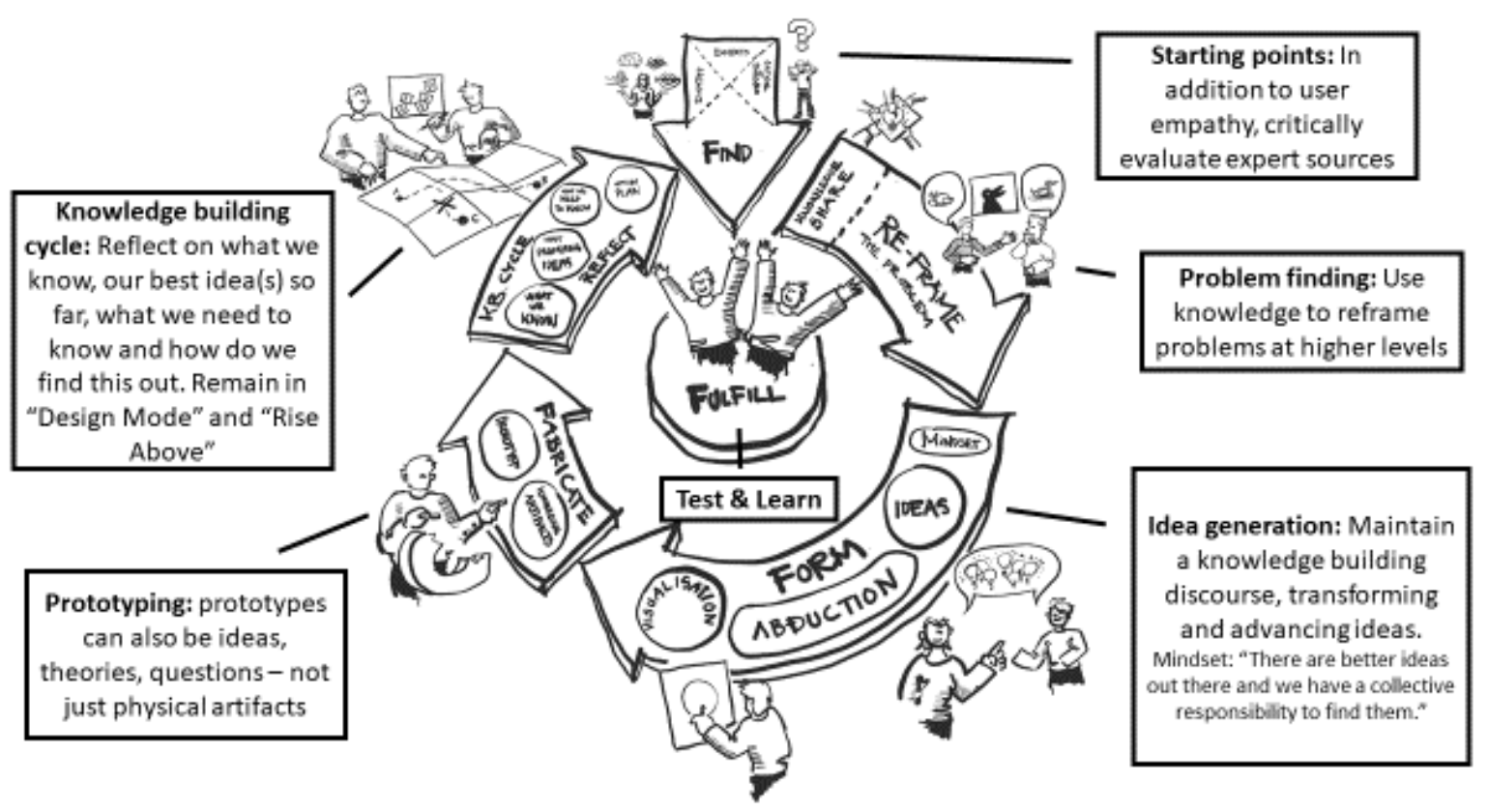

Figure 1. An integrated model - a design thinking process combined with knowledge
building principles

Thirdly, conducting a design thinking workshop is relatively straightforward, there is a wealth of "how to" information available in terms of literature, blogs, videos, manuals etc. Knowledge building requires more from facilitators (and students), in terms of maintaining the knowledge building discourse and using "scaffolding" questions ${ }^{1}$ to close gaps in knowledge. While, in our experience as a design school, creativity comes naturally to most student teams, the collaborative sustained development of ideas does not and requires encouragement. To facilitate this, we produced an instructional animation film and a "knowledge building canvas" which is in the form of a template consisting of the essential building blocks of knowledge building. ${ }^{2}$ We also developed an instructional exercise, "Solving Titanic Problems with Creativity and Knowledge". Teams are placed, figuratively, "on deck" one minute after HMS

\footnotetext{
${ }^{1}$ Scaffolding questions include: "How might this idea be tested?" "What do you still need to find out?" "How do you know this idea will solve the problem?" "What might be improved?"

${ }^{2}$ The building blocks of knowledge building on the canvas are: understanding user needs, critique of the statusquo, expert sources of information, what we know now, our most promising ideas, what do we need to know? Action plan to close knowledge gaps.
} 
Titanic struck the iceberg at 23:40 (ship's time) on $14^{\text {th }}$ April 1912, and asked to develop ideas that may have saved more passengers and crew than the 705 that survived the tragic sinking. This challenge requires both creativity and knowledge to succeed and introduces teams to the principles of knowledge building [15].

\section{STUDIES WITH THE COMBINED APPROACH}

We have carried out a further series of randomized control trials again using several hundred students working in multidisciplinary teams. In these studies, the "design thinking" teams made up the control group and teams using the combined design thinking/knowledge building approach made up the test group. Our findings are that the concepts from the "combined approach" teams are more innovative and more elaborate in terms of detail than the concepts from teams using design thinking alone. For example, in another study, involving 70 design and business students, teams were asked to develop a range of ideas that might assist a western European family reduce their environmental footprint in terms of their household laundry practices. Student teams were asked to produce ideas in two categories, firstly, what we termed "hygiene" factors, things that could be implemented with existing technology and would fit in easily with current life-styles or requiring modest changes in current habits. The teams were also invited to suggest more innovative ideas that may require a combination of technologies from other areas or significant changes in behaviour and lifestyle. Teams were asked to calculate and document any savings their ideas would make in term of energy, $\mathrm{CO}_{2}$ emissions, water and chemicals used etc. The resulting concepts were evaluated by an independent panel of three experts with no knowledge of the team selection criteria embedded in this study. The design thinking teams generated a range of ideas but these were largely "basic" in nature: don't tumble dry or iron clothes, use shorter washing cycles, only wash full loads, wash at 30 degrees, use the correct amount of detergent, hand wash, read care labels etc. The teams using the combined approach also generated similar ideas but several additional ones, wear self-cleaning fabrics, (i.e. wool), various re-cycling ideas, the use of filter bags to recycle microfibers, use natural even "home-made soaps, freeze, steam clean or "air-wash" clothes, consider community washing etc. This demonstrated higher knowledge and translated into more elaborate concepts. In terms of the "innovative concepts", the panel rated these concepts numerically in terms of concept fluency, flexibility, originality, elaboration and relevance. This allowed the panel to evaluate the concepts in terms of degree of innovation, (Table 2):

Table 2. Comparison of design thinking teams (control group) and design thinking/knowledge building teams (test group)

\begin{tabular}{|l|l|l|l|l|l|}
\hline Innovation Level & Very Low & Low & Medium & High & Very high \\
\hline Design thinking teams & 1 & 1 & 4 & 1 & 0 \\
\hline Design thinking/knowledge building teams & 0 & 0 & 2 & 3 & 2 \\
\hline
\end{tabular}

The design thinking teams generated some interesting ideas: "smart washing machines", educational apps, a "dirty ball" that attracts dirt, and use of solar panels. The concepts from the teams using the combined approach included: quite sophisticated "smart" washing machines, the use of nanotechnology in fibre manufacture and regular use, the incorporation of silver fibres into clothing, the application of directional layered textiles with asymmetric filaments inspired by biomimicry and an "Eco" washing service. In general these teams concepts were more flexible, (contained more idea types), original and more elaborate containing more detail and documented savings. Immediately following the study, all students were asked individually to rate which stages of the model were most (or least) useful in solving the challenge using a simple points system: 0 points - not at all useful, 1 point - slightly useful, 2 points - useful, 3 points - very useful. The design thinking model scored 212 points, and the combined model 354 points. Even accounting for the fact that the combined model has an extra segment to attract votes, (the knowledge building cycle - figure 1), if this score is subtracted the overall scores for the combined model were $25 \%$ higher than for the design thinking model suggesting that, from a student perspective, the combined model was more useful. 


\section{CONCLUSIONS AND FURTHER RESEARCH}

Empirical studies have revealed that, for problems that require creativity and knowledge to solve them, integrating knowledge building principles within a design thinking framework enables multidisciplinary student teams to develop more innovative and elaborate design concepts. This suggests subsequent knowledge acquisition assists innovative concept development. Combining the design thinking and knowledge building paradigms does require a set of pedagogical aids in the form of models, templates and exercises to communicate the core concepts and the value they can bring to creative problem solving.

\section{REFERENCES}

[1] Rahman, M. (2019). 21st Century Skills, "Problem Solving” Defines the Concept. Asia Journal of Inter-disciplinary Research. V2 pp 71-81 2019.

[2] Shay, S. and Steyn, D. (2015). Enabling Knowledge Progression in Vocational Curricula. In Knowledge-building Educational Studies in Legitimation Code Theory. Maton, K., Hood, S. \& Shay, S. (eds.). Routledge.

[3] Kimbell, L. (2011). Rethinking design thinking: Part 1. Design and Culture, 3(3), 285-306.

[4] Norman, D. (2020). Design Thinking: A useful Myth. Core 77 [online]. Available at: https://www.punyamishra.com/wp-content/uploads/2017/09/02.-Norman-DesignThinking_-A-Useful-Myth-.pdf [Accessed on 2010, 5 June].

[5] Norman, D. A. and Verganti, R. (2012). Incremental and Radical Innovation: Design research versus technology and meaning change. Design Issues 30(1), 78-96.

[6] Nilsson, P. (2012). Taxonomy of Creative Design. Sense and Sensation. http://www.senseandsensation.com/2012/03/taxonomy-of-creative-design.html. Accessed on 18/05/2016.

[7] Guilford, J. P. (1988). Some Changes in the Structure of the Intelligent Model. Educational and Psychological Measurement, 48, 1-4.

[8] McElheron, P. J. (2017). Building on Design Thinking: The contribution of creativity \& knowledge. A study of two innovation workshops. Cumulus REDO Conference. Kolding, Denmark. 30 May-3 June 2017.

[9] Reiter-Palmon, R., Ben, W. and de Vreede, T. (2011). Team Creativity and Innovation: The effect of team composition, society, process and cognition. In: Handbook of organizational Creativity. Ed. Mumford, M. Academic Press.

[10] Kelly, T \& Kelly, D. Creative Confidence. Available at: https://www.creativeconfidence.com/CreativeConfidence_preandintro.pdf. Accessed, 20197 January.

[11] Engle, R. A. and Conant, F. R. (2002). Guiding principles for productive disciplinary engagement: Explaining an emerging argument in a community of learners in a classroom. Cognition \& Instruction20, 399-483.

[12] Bereiter, C. and Scardamalia, M. (2003). Learning to Work Creatively with Knowledge. In De Corte, E., Verschaffel, L, Entwistle, N. \& Merriënboer, J. van (Eds.). Unravelling basic components and dimensions of powerful learning environments. EARLI Advances in Learning Instruction Series.

[13] Scardamalia, M. and Bereiter, C. (2006). Knowledge Building: Theory, pedagogy, and Technology.

In K. Sawyer (Ed.). Cambridge Handbook of Learning Sciences (pp. 97-118).

[14] Scardamalia, M. and Bereiter, C, (2017). Two Modes of Thinking in Knowledge Building. Revista Catalana de Pedagogia, 12 (2017) p 61-83.

[15] McElheron, P. J. (2018). Building on Design Thinking: pedagogical challenges and Titanic solutions. Knowledge Building Summer Institute Conference. Toronto, Canada. 14-17 August 2018. 